\title{
Discrete truncated Wigner approach to dynamical phase transitions in Ising models after a quantum quench
}

\author{
Reyhaneh Khasseh, ${ }^{1,2}$ Angelo Russomanno, ${ }^{3}$ Markus Schmitt, ${ }^{4}$ Markus Heyl, ${ }^{3}$ and Rosario Fazio ${ }^{1,5}$ \\ ${ }^{1}$ Abdus Salam ICTP, Strada Costiera 11, I-34151 Trieste, Italy \\ ${ }^{2}$ Department of Physics, Institute for Advanced Studies in Basic Sciences (IASBS), Zanjan 45137-66731, Iran \\ ${ }^{3}$ Max-Planck-Institut für Physik Komplexer Systeme, Nöthnitzer Straße 38, D-01187, Dresden, Germany \\ ${ }^{4}$ Department of Physics, University of California at Berkeley, Berkeley, California 94720, USA \\ ${ }^{5}$ Dipartimento di Fisica, Università di Napoli Federico II, Monte S. Angelo, I-80126 Napoli, Italy
}

(Received 29 April 2020; revised 17 June 2020; accepted 17 June 2020; published 6 July 2020)

\begin{abstract}
By means of the discrete truncated Wigner approximation, we study dynamical phase transitions arising in the steady state of transverse-field Ising models after a quantum quench. Starting from a fully polarized ferromagnetic initial condition, these transitions separate a phase with nonvanishing magnetization along the ordering direction from a disordered symmetric phase upon increasing the transverse field. We consider two paradigmatic cases, a one-dimensional long-range model with power-law interactions $\propto 1 / r^{\alpha}$ decaying algebraically as a function of distance $r$ and a two-dimensional system with short-range nearest-neighbor interactions. In the former case, we identify dynamical phase transitions for $\alpha \lesssim 2$ and we extract the critical exponents from a data collapse of the steady-state magnetization for up to 1200 lattice sites. We find identical exponents for $\alpha \lesssim 0.5$, suggesting that the dynamical transitions in this regime fall into the same universality class as the nonergodic mean-field limit. The two-dimensional Ising model is believed to be thermalizing, which we also confirm using exact diagonalization for small system sizes. Thus, the dynamical transition is expected to correspond to the thermal phase transition, which is consistent with our data upon comparing to equilibrium quantum Monte Carlo simulations. We further test the accuracy of the discrete truncated Wigner approximation by comparing against numerically exact methods such as exact diagonalization, tensor network, as well as artificial neural network states and we find good quantitative agreement on the accessible time scales. Finally, our work provides an additional contribution to the understanding of the range and the limitations of qualitative and quantitative applicability of the discrete truncated Wigner approximation.
\end{abstract}

DOI: 10.1103/PhysRevB.102.014303

\section{INTRODUCTION}

Recent impressive developments underline the rich phase structures that can be generated by forcing isolated quantum matter out of equilibrium. Some examples of these phenomena are the emergence of exotic phases, loss of adiabaticity across critical points in the context of the Kibble-Zurek mechanism, and nonequilibrium phase transitions. These are some of the multiple aspects currently at the center of an intense theoretical and experimental activity, as summarized in the reviews [1-7].

A paradigmatic protocol to drive a many-body system out of equilibrium, routinely used in experiments and intensively studied theoretically, is a quantum quench. After initializing the system in a state that can be thought of as a ground

Published by the American Physical Society under the terms of the Creative Commons Attribution 4.0 International license. Further distribution of this work must maintain attribution to the author(s) and the published article's title, journal citation, and DOI. Open access publication funded by the Max Planck Society. state of a given initial Hamiltonian, it is allowed to evolve after an abrupt change of a Hamiltonian parameter. The longtime steady states after such quantum quenches can feature symmetry-broken phases and singular behavior at the transition toward the disordered phase. These dynamical phase transitions (DPTs) [8-10] may be understood as transitions in the microcanonical ensemble in case the many-body system thermalizes, driven by shifting the system's energy across the symmetry-restoration threshold. In nonergodic systems, however, long-time steady states can be realized which cannot be described in terms of the conventional thermodynamic ensembles. As a particular consequence, such systems allow the generation of phases and phase transitions with properties that cannot be realized in any equilibrium context [11].

In this paper, we focus on DPTs realized in spin-1/2 Ising models in transverse fields. We consider the case of longrange interacting models, which have recently attracted a lot of attention [8,10,12-20] and constitute a paradigmatic class of nonergodic systems capable of generating nonequilibrium steady states as reported both theoretically [8-10,21] and experimentally [22-24]. It was shown $[8,10]$ that starting from an initial fully polarized state along the ordering direction, the asymptotic state of these systems can undergo a transition 
from an ordered phase at small fields to a disordered one when the field exceeds a critical value. While inherently of nonequilibrium character, the resulting phases can be characterized by means of the conventional Landau paradigm via local order parameters. Still, the understanding of the nature of the transition between the ordered and disordered phases has remained limited. In particular, it is unclear to which extent these DPTs follow the general paradigm of continuous equilibrium transitions such as whether they can be categorized in terms of universality classes and therefore whether the concepts of universality and scaling extend to this nonequilibrium dynamical regime. We remark that here we completely neglect the analysis of singular behaviors in the (infinite-size) time dynamics, which is another aspect of DPTs [5] with some connection with the symmetry-breaking behavior [10].

In this paper, we show that the DPTs after quantum quenches in transverse-field Ising chains with power-law decaying interactions $\left(\sim r^{-\alpha}\right)$ can feature scale invariance. We find evidence that the critical exponents of the DPT are universal over a large range of interaction exponents $\alpha$. Via finite-size scaling of the time-averaged longitudinal magnetization, we identify the critical value of the field $h_{c}$ of the DPT and, in particular, determine the scaling exponents of the transition. By studying the decay in time of the longitudinal magnetization, we are able to put bounds to the values of $\alpha$ above which the ordered phase disappears. We can confirm the existence of two phases as long as $\alpha \lesssim 2$. The time-averaged magnetization decreases with the averaging time and never reaches a plateau for values of $\alpha$ larger or equal than 2 . This suggests that only the trivial phase survives in this regime of $\alpha$, consistent with previous works [10].

For $\alpha=0$, the dynamics can be solved via an effective mean-field description, which becomes exact in the thermodynamic limit (see, for example, Ref. [25]). For $\alpha \geqslant 0$, we compute the quantum real-time evolution by means of the discrete truncated Wigner approximation (DTWA) [26]. It has already been reported that DTWA compares well with other methods for long-range models $[14,27]$ and, as we are going to show, also works very well for our problem, giving a very good comparison with the results of a recent numerical study using tensor network methods [10]. The DTWA has the advantage that it allows us to access large sizes with moderate computational resources polynomially scaling in system size. Consequently, we can also perform finite-size scaling in longrange systems where it is crucial to reach large system sizes to tell the difference from the infinite-range $(\alpha=0)$ case.

When analyzing scale invariance at the DPT, we find that the DTWA gives rise to scaling exponents identical to the mean-field ones at $\alpha=0$. For finite $\alpha$, at the mean-field level, the exponents are of course independent on the range of the interaction. This is different for the DTWA, it compares well with exact methods, as emphasized above, and it is able to capture correlations. Therefore, in principle, it can give reliable scaling exponents. We computed the dependence on $\alpha$ of the scaling exponents of the magnetization, and observed a significant deviation from the mean-field values at $\alpha \sim 1$. As discussed in the relevant sections, in this regime of $\alpha$ DTWA is not able to achieve accurate precision for a reliable scaling. It clearly indicates, however, when the deviations from mean field occur.

The favorable scaling of the DTWA with the number of sites allows us to also tackle the study of the DPT in higher dimensions, a problem not touched so far in literature. As long as the spins are interacting via long-range exchange couplings, we do not expect significant dependence on the dimensionality. This is why we decided to study a two-dimensional system with nearest-neighbor (NN) coupling. Also, in this case, we expect a DPT. Here, however, the critical behavior should clearly deviate from the mean-field case. In this case, we can only compare to exact diagonalization at small system sizes to test the quality of the DTWA approach. As we will show in the second part of the paper, we are able to detect the existence of the DPT through an analysis of the magnetization and of the Binder cumulant. We perform a comparison with finitetemperature quantum Monte Carlo results and we see that this DPT corresponds to the thermal transition. We show that this result is physically sound because the model is quantum chaotic. We find additional support for this conclusion by using exact diagonalization and showing that the level-spacing statistics is Wigner-Dyson.

In addition, we believe that our work may also contribute to a better understanding of the range and the limitations of qualitative and quantitative applicability of the DTWA. The DTWA has proved to work better in the context of longrange interactions [27]. The reason is that DTWA catches the long-distance quantum correlations only partially and then works better when the model is near infinite range. The situation is similar to the one of the mean-field approximation, with the improvement that here quantum correlations are taken into account at least partially, giving rise to scaling exponents beyond the mean-field result. In the twodimensional case, quantum correlations become more relevant for the dynamics and we find that the DTWA provides only a qualitative (but remarkably meaningful) description for the dynamics.

The paper is organized as follows. In Sec. II, we introduce the model and also define the order parameter for the phase transition. In Sec. III, we discuss the DTWA theory in detail and we show how to apply it to our model. In Sec. III B, we compare the DTWA approach for this model with known results both in the infinite-range interaction case-where exact diagonalization is also possible for large sizes-and long-range interaction where the time-dependent variation principle (TDVP) method is used. For the range of parameters we are interested in, we find that the comparison is very good. In Sec. IV A, we perform the finite-size scaling analysis for the one-dimensional long-range case. We first consider the case $\alpha=0$ where we compare with the exact diagonalization results and find that the comparison is very good. Then we move to analyze the case $\alpha \neq 0$ and see that the transition exists only when $\alpha \lesssim 2$. The results for the short-range two-dimensional models are discussed in Sec. IV B. Finally, Sec. V is devoted to the conclusions and further perspectives. The Appendices contain additional details of the numerical analysis. 


\section{THE MODEL}

As anticipated in the Introduction, we will study a system of $N$ interacting spins governed by the Hamiltonian

$$
\hat{H}=-\sum_{i \neq j} J_{i j} \hat{\sigma}_{i}^{x} \hat{\sigma}_{j}^{x}-h \sum_{i} \hat{\sigma}_{i}^{z},
$$

where $\hat{\sigma}_{j}^{x, z}$ are the Pauli matrices of the spin located in the $j$ th site, $h$ is an external transverse field, and $J_{i j}$ is the exchange coupling between the spins. We will consider two cases (assuming to express the energies in units of the exchange coupling):

(1) A long-range interacting spin exchange,

$$
J_{i j}=\frac{K_{\alpha}}{r_{i j}^{\alpha}},
$$

in one dimension. We assume periodic boundary conditions and define the distance between two sites as $r_{i j}=\min [\mid i-$ $j|, N-| i-j \mid]$. The Kac factor [28] $K_{\alpha}$ is defined as $K_{\alpha}^{-1} \equiv$ $\frac{1}{N-1} \sum_{i \neq j}^{N} r_{i j}^{-\alpha}$ and ensures that the Hamiltonian is extensive.

(2) A short-range interacting spin on a $d$-dimensional cubic lattice where the exchange coupling

$$
J_{i j}=\frac{1}{d} \delta_{i, \mathrm{NN}_{(j)}}
$$

is different from zero $\left(\delta_{l, m}\right.$ is the Kronecker delta) only if $i$ and $j$ are NNs. We will assume periodic boundary conditions and consider the cases of $d=1$ and $d=2$ (a square lattice of size $L, N=L^{2}$ ).

The system is initialized in the state fully polarized along $x$ :

$$
\left|\psi_{0}\right\rangle=\bigotimes_{i}|\rightarrow\rangle_{i}
$$

We then perform a quantum quench with the dynamics governed by the Hamiltonian of Eq. (1). We are interested in the evolution of the total $x$ (longitudinal) magnetization which is given by

$$
m_{x}(t)=\frac{1}{N} \sum_{i=1}^{N}\left\langle\psi(t)\left|\hat{\sigma}_{i}^{x}\right| \psi(t)\right\rangle,
$$

and the order parameter for the DPT is the long-time average of this magnetization:

$$
\bar{m}_{x}=\lim _{T \rightarrow \infty} \frac{1}{T} \int_{0}^{T} d t m_{x}(t) .
$$

(We will always use the finite- $T$ version of this quantity, $\bar{m}_{x}(T)$. We will not specify the dependence on $T$ in those cases where we have attained convergence.) In the short-range twodimensional case, we will also analyze the Binder cumulant in the long-time limit, defined as

$$
U_{L}=1-\overline{\frac{m_{x}^{(4)}}{3\left(m_{x}^{(2)}\right)^{2}}},
$$

where we defined $m_{x}^{(l)}(t)=\frac{1}{N^{l}}\left\langle\psi(t)\left|\left[\sum_{i=1}^{N} \hat{\sigma}_{i}^{x}\right]^{l}\right| \psi(t)\right\rangle$.

The Binder cumulant is a measure for non-Gaussian fluctuations of the order parameter. At equilibrium in the thermodynamic limit, it acquires two different universal values in two phases: The Gaussian value 0 in the disordered phase and the value $2 / 3$ in the ordered phase. At the transition point, the Binder cumulant is scale invariant and it is a very convenient numerical probe for the existence of an equilibrium transition [29]. We will also show that in this nonequilibrium context for the 2D short-range case, it behaves in the same way and allows us to probe the existence of a transition.

\section{DISCRETE TRUNCATED WIGNER APPROXIMATION}

Before getting into the discussion of the results, it is useful to recap the basic ideas behind the DTWA and to discuss the accuracy of this method for this problem. In the following, we first review methodological details of the DTWA and afterward we use exact diagonalization and matrix product state descriptions by means of a time-dependent variation principle (MPS-TDVP) data for a quantitative comparison.

\section{A. DTWA method}

The DTWA is a semiclassical approximation, which has been used in many contexts concerning long-range interacting spin systems and has given noteworthy results, in terms of comparison with exact results and scalability to large system sizes. Precise details on the background can be found in Refs. [14,27]; here we outline our concrete implementation. All the analysis is based on the construction of the discrete Wigner representation [26] which is a generalization to discrete Hilbert space of the usual Wigner representation (details can be found in Ref. [30]). Summarizing, Wootters has shown that, given a discrete Hilbert space, the quantum dynamics can be represented through a discrete basis of operators. In the case of a single $1 / 2$ spin, a possible basis choice is

$$
\hat{A}_{\beta}=\frac{\mathbf{1}+\mathbf{s}_{\beta} \cdot \hat{\boldsymbol{\sigma}}}{2}
$$

where $\boldsymbol{s}_{\beta}$ can take the values $\left(\begin{array}{lll}1 & 1 & 1\end{array}\right),\left(\begin{array}{lll}-1 & 1 & -1\end{array}\right)$, $\left(\begin{array}{lll}1-1 & -1\end{array}\right)$, and $\left(\begin{array}{lll}-1 & -1 & 1\end{array}\right)$ and $\hat{\sigma}=\left(\begin{array}{lll}\hat{\sigma}^{x} & \hat{\sigma}^{y} & \hat{\sigma}^{z}\end{array}\right)$.

With this basis choice, the expectation of any operator $\hat{\mathcal{O}}$ acting on the Hilbert space of the single spin can be written as

$$
\langle\mathcal{O}\rangle_{t}=\sum_{\beta} w_{\beta} \mathcal{O}_{\beta}(t),
$$

where $w_{\beta} \equiv \frac{1}{2} \operatorname{Tr}\left[\hat{A}_{\beta} \hat{\rho}\right]$ is the Wigner function, $\mathcal{O}_{\beta}^{w}(t)=$ $\frac{1}{2} \operatorname{Tr}\left[\hat{A}_{\beta} \hat{\mathcal{O}}(t)\right]$ are the Weyl symbols, and $\hat{\mathcal{O}}(t) \equiv \mathrm{e}^{i \hat{H} t} \hat{\mathcal{O}} \mathrm{e}^{-i \hat{H} t}$. This representation can also be extended to our case of $N$ spins considering as basis operators

$$
\hat{A}_{\beta}=\hat{A}_{\beta_{1}} \otimes \hat{A}_{\beta_{2}} \otimes \hat{A}_{\beta_{3}} \otimes \cdots \otimes \hat{A}_{\beta_{N}}
$$

and writing as before the expectation of any operator $\hat{\mathcal{O}}$ acting on the Hilbert space of the $N$ spins as

$$
\langle\mathcal{O}\rangle_{t}=\sum_{\beta} w_{\beta} \mathcal{O}_{\beta}(t) .
$$

Up to now, everything is exact. The DTWA amounts to approximate the time-evolved basis operators as factorized objects,

$$
\hat{A}_{\beta}(t)=\mathrm{e}^{-i \hat{H} t} \hat{A}_{\beta} \mathrm{e}^{i \hat{H} t} \simeq \hat{A}_{\beta_{1}}(t) \otimes \hat{A}_{\beta_{2}}(t) \otimes \cdots \otimes \hat{A}_{\beta_{N}}(t),
$$


where

$$
\hat{A}_{\beta_{j}}(t)=\frac{\mathbf{1}+s_{j, \beta_{j}}^{x}(t) \hat{\sigma}_{j}^{x}+s_{j, \beta_{j}}^{y}(t) \hat{\sigma}_{j}^{y}+s_{j, \beta_{j}}^{z}(t) \hat{\sigma}_{j}^{z}}{2} .
$$

The $s_{\beta_{j}}^{\nu}(t)$ are initialized with the value for the corresponding $\beta_{j}$ given in Eq. (8) and obey a simple classical Hamiltonian dynamics given by

$$
\dot{s}_{j, \beta_{j}}^{\mu}(t)=\left\{s_{j, \beta_{j}}^{\mu}(t), \mathcal{H}\right\}=2 \sum_{\nu \rho} \epsilon_{\mu \nu \rho} s_{j, \beta_{j}}^{\rho}(t) \frac{\partial \mathcal{H}}{\partial s_{j, \beta_{j}}^{\nu}} .
$$

Here the symbol $\{\cdots, \cdots\}$ is the Poisson bracket, $\epsilon_{\mu \nu \rho}$ is the Levi-Civita fully antisymmetric tensor, the variables $s_{j, \beta_{j}}^{\mu}$ obey the angular-momentum Poisson brackets $\left\{s_{j, \beta_{j}}^{\mu}, s_{l, \beta_{l}}^{v}\right\}=$ $\delta_{j l} \epsilon_{\mu \nu \rho} s_{j, \beta_{j}}^{\rho}$, and the classical effective Hamiltonian is defined as

$$
\mathcal{H}\left(\left\{s_{j, \beta_{j}}^{\mu}\right\}\right)=-\sum_{i \neq j}^{N} J_{i j} s_{i, \beta_{i}}^{x} s_{j, \beta_{j}}^{x}-h \sum_{i} s_{i, \beta_{i}}^{z} .
$$

For instance, the total longitudinal magnetization Eq. (5) can be evaluated in the DTWA scheme as

$$
m_{x}(t)=\sum_{\beta} w_{\beta} \frac{1}{N} \sum_{i=1}^{N} s_{i, \beta_{i}}^{x}(t) .
$$

In this form, it still unpractical from the numerical point of view because the index $\beta$ runs over $4^{N}$ values, so the sum would be unfeasible for large system sizes. The solution comes from the relation $\sum_{\beta} w_{\beta}=1$, so, in the cases when $w_{\beta} \geqslant 0$, it behaves as a probability distribution and it can be sampled through Monte Carlo sampling. With the initialization we choose, we are in one of these lucky cases (see Ref. [27] for more details) and we can write Eq. (16) as the average over $n_{r}$ random initializations where each $s_{j, \beta_{j}}$ is initialized with probability $1 / 2$ in the condition $\left(\begin{array}{lll}1 & 1 & 1\end{array}\right)$ and probability $1 / 2$ in the condition $(1-1-1)$.

We remark that this operation is a sample over an operator basis. Indeed, the initial density matrix can be written as

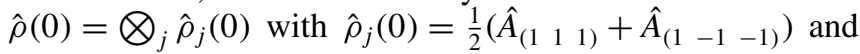
the two operators $\hat{A}_{\left(\begin{array}{lll}1 & 1 & 1\end{array}\right)}$ and $\left.\hat{A}_{(1-1}-1\right)$ are sampled with equal $1 / 2$ probability. Many possible choices of operator bases are possible, moving to each of these different representations by means of a unitary transformation. We provide an example of that in Appendix A.

Remarkably, the error bars do not scale with the system size, so this method is feasible also in the case of large systems. Moreover, results converge with a small number of randomness realizations $\left(n_{r}\right)$; we show an example of this convergence in Appendix A. Unless otherwise specified here, we use $n_{r}=504$.

Finally, different sampling schemes, related to different choices of the operator in Eq. (8), can be employed. In Appendix A, we briefly discuss these possible choices. All the results presented in the paper are essentially independent of the sampling method. Unless we specify otherwise, throughout the paper we use the sampling scheme specified in Eq. (8).

In the following, we are going to compare the DTWA method with the results of other numerical methods to also show its value in our case.

\section{B. Comparison with other methods}

The comparison was done only in the case of onedimensional power-law interaction. In this case, in addition to the possibility to have results from exact diagonalization, it is possible to compare our data with tensor-network (the MPS-TDVP) results [10,31] for larger sizes.

First, we consider the case $\alpha=0$ of infinite-range interactions. In this limit, the model reduces to the Lipkin-MeshkovGlick model whose exact diagonalization (ED) dynamics can be easily studied. With all the site-exchange operators conserved, there is a superextensive number of constants of motion and the dynamics becomes integrable. Thanks to the conservation of the modulus of the total spin, the quantum dynamics is restricted to a Hilbert subspace whose dimension scales linearly with the system size, making the solution of large system sizes feasible. Specifically, the Hamiltonian commutes with the total-spin operator $\hat{\mathbf{S}}^{2}\left[\hat{\mathbf{S}}=\frac{1}{2} \sum_{j} \hat{\boldsymbol{\sigma}}_{j}\right.$ with $\left.\hat{\boldsymbol{\sigma}}_{j} \equiv\left(\begin{array}{lll}\hat{\sigma}_{j}^{x} & \hat{\sigma}_{j}^{y} & \hat{\sigma}_{j}^{z}\end{array}\right)^{T}\right]$ and we can restrict to the $\hat{\mathbf{S}}^{2}$-subspace with eigenvalue $S(S+1)$ with $S=N / 2$, which has a dimension $N+1$. (For a detailed explanation, see, for instance, Ref. [32]). We show some instances of comparison in Fig. 1. Let's first consider the case $N=100$. We see that the curves of $m_{x}(t)$ deviate quite soon from each other, both for $h<1$ and $h>1$, but the time average (the one we are interested in) is actually the same (it is marked in the plots by a dashed horizontal line). Dynamics up to a time $t \sim 30$ is quantitatively correct. For larger times, the quantum revivals are not captured properly. This feature, however, shifts to larger times upon increasing system size. Thus, for large systems this discrepancy becomes less and less relevant, making a description via the DTWA more accurate.

We also show results for $N=10$. Here we can see that in the ED case, a phenomenon appears which is not captured by DTWA, the Rabi oscillations. Indeed, in this system an extensive number of eigenstates breaks the $\mathbb{Z}_{2}$ symmetry in the thermodynamic limit. For any finite size, the true eigenstates are the even and odd superposition of these symmetrybreaking states and are separated by an exponentially small gap. Preparing the system in a symmetry-breaking state [as the one in Eq. (4)] therefore gives rise to Rabi oscillations of the magnetization with a frequency equal to the gap. Because this gap is exponentially small in the system size, we cannot see these oscillations in Fig. 1(a), where the size is $N=100$ and the gap is negligibly small $\left(\sim \mathrm{e}^{-100 \ln (1 / 0.32)}\right)$. But we can see them in Fig. 1(c) and they are not caught by DTWA.

The existence of the Rabi oscillations is intimately related to the existence of a $\mathbb{Z}_{2}$ symmetry and the presence of resonant symmetry-breaking states put in interaction by the term with the $h$ field. Explicitly breaking the symmetry breaks the resonance and there are no more oscillations. We do this in Fig. 1(c) where we also show a curve of $m_{x}(t)$ obtained, adding to the Hamiltonian a small symmetry-breaking term $\delta h \sum_{j} \hat{\sigma}_{j}^{x}$. We see that there are no Rabi oscillations and the comparison with DTWA in terms of average is very good. So, in some sense, in DTWA one implicitly adds to the Hamiltonian a small symmetry-breaking term. This is just what we operatively do when we want to see a quantum phase transition. We add a small symmetry-breaking term, we go to the thermodynamic limit, and then we send the small 

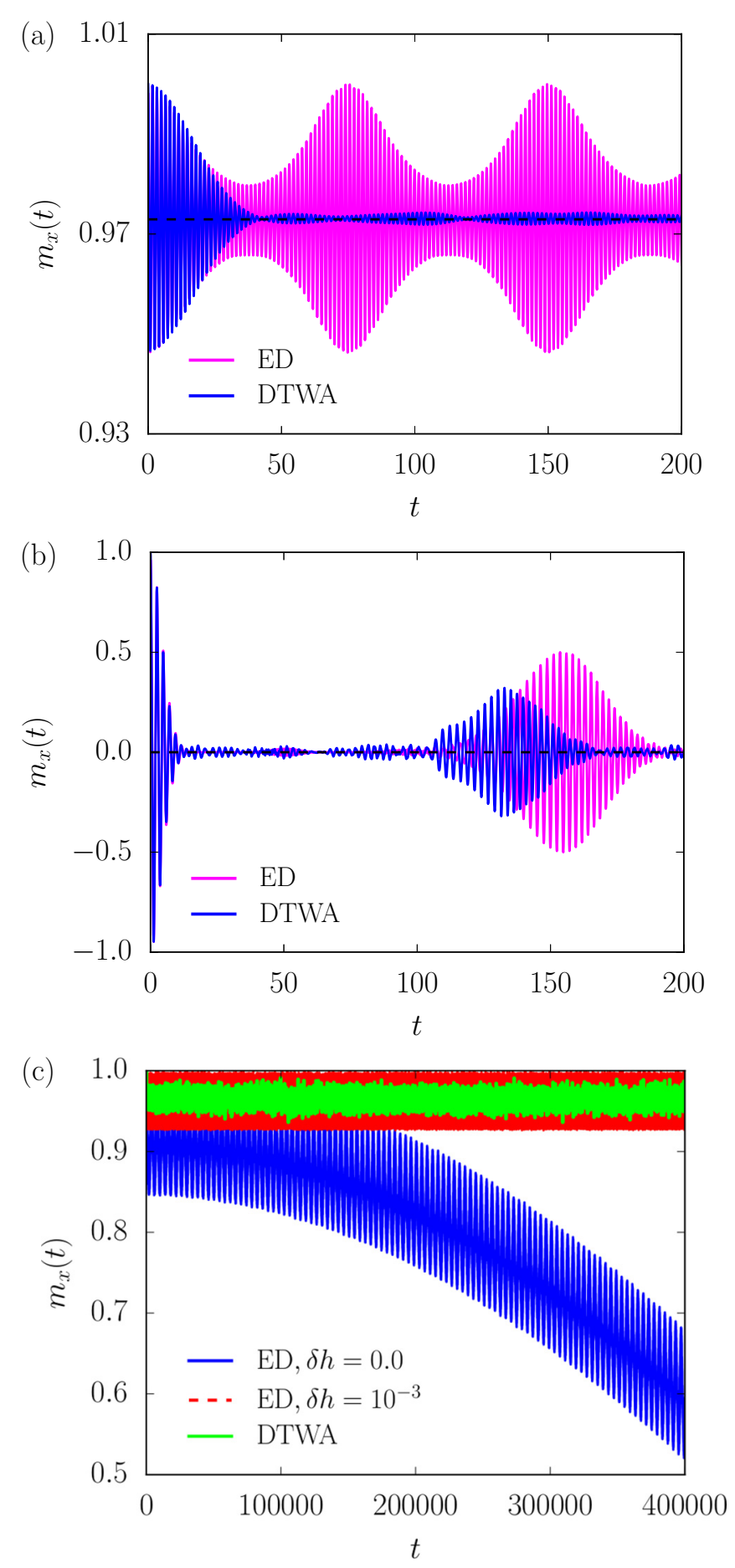

FIG. 1. Comparison of DTWA method with ED for different parameters. In panel (a) we take $N=100$ and $h=0.32$, in panel (b) we take $N=100$ and $h=1.5$, in panel (c) we use $N=10$ and $h=0.32$. In panels (a) and (b) we can see that the ED and DTWA curves loose agreement after a while but their time averages coincide (dashed horizontal line). DTWA cannot catch the Rabi oscillations; they are eliminated by adding a small symmetry-breaking field in the ED case [see (c))]. In (a), we use the sampling scheme specified by Eq. (A1) while in the other panels we use the sampling scheme given by Eq. (8).
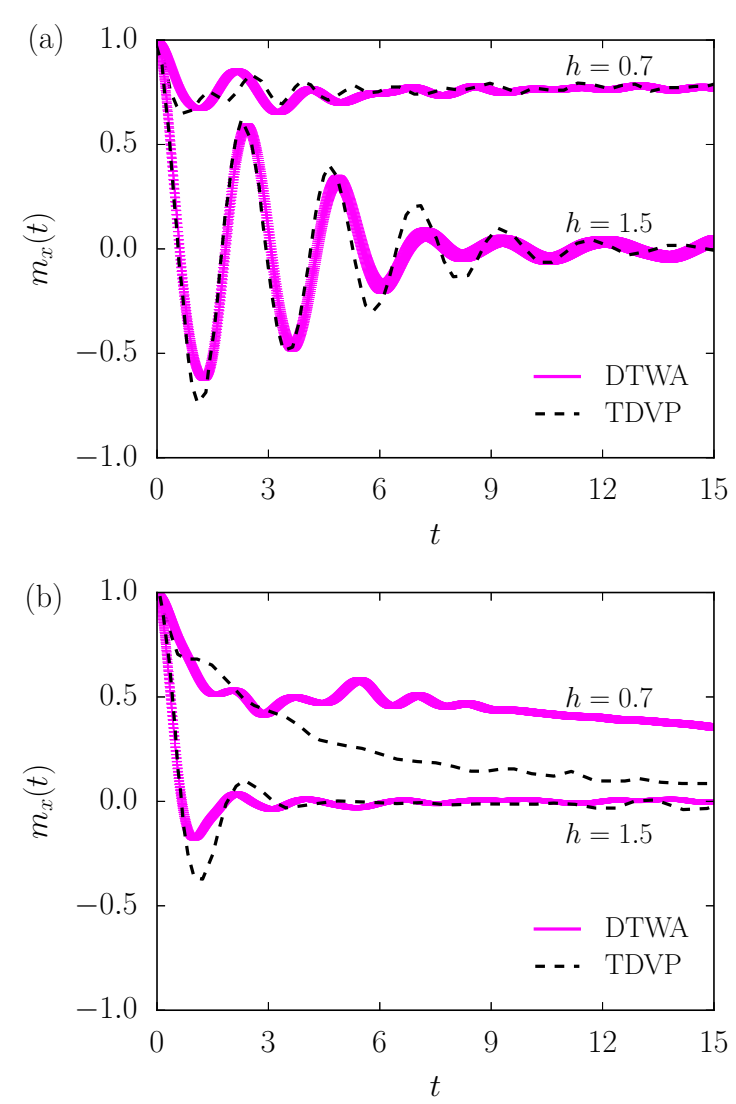

FIG. 2. The instantaneous magnetization $m_{x}(t)$ versus $t$ : Comparison of the results obtained with DTWA and TDVP [10] methods for different values of $\alpha$ and $h$. In panel (a) we consider $\alpha=1.5$ and in panel (b) we consider $\alpha=3.0$. As expected from Ref. [27], we see a much better agreement at smaller $\alpha$. Other parameters: $N=100$.

symmetry-breaking term to zero. Because we are interested here in the existence of a dynamical quantum phase transition with $\mathbb{Z}_{2}$ symmetry breaking, this is exactly what we should do. DTWA does this implicitly for us, and in the thermodynamic limit the presence of a small symmetry-breaking term makes no difference both for DTWA and ED.

For $\alpha \neq 0$, we can compare our DTWA results for the transverse magnetization $m_{x}(t)$ with the corresponding ones obtained through the TDVP method [10,31] (see Fig. 2) for the case of $N=100$ sites. The timescales we consider are much shorter than the times exponential in $N$ needed for seeing the Rabi oscillations. Let us start focusing on the case $\alpha=1.5$ [Fig. 2(a)]. We see that in this case the agreement is quite good both inside the symmetry-breaking phase $(h=0.7)$ and outside it $(h=1.5)$. On the opposite, for $\alpha=3$ [Fig. 2(b)], the agreement is very good only when $h=1.5$. When $h=0.7$, the DTWA result decays much more slowly than TDVP. The two methods are in agreement for small values of $\alpha$, as we expected from the existing literature on DTWA. To show the very good agreement when $\alpha$ is small, we plot in Fig. 3 the time-averaged longitudinal magnetization $\bar{m}_{x}$ versus $h$ for $\alpha=0.1$ and $\alpha=1.5$ obtained through the two methods. In both cases, we see a very good agreement between the two methods. So, in the small- $\alpha$ regime we are interested in, the DTWA compares very well with the known results obtained 

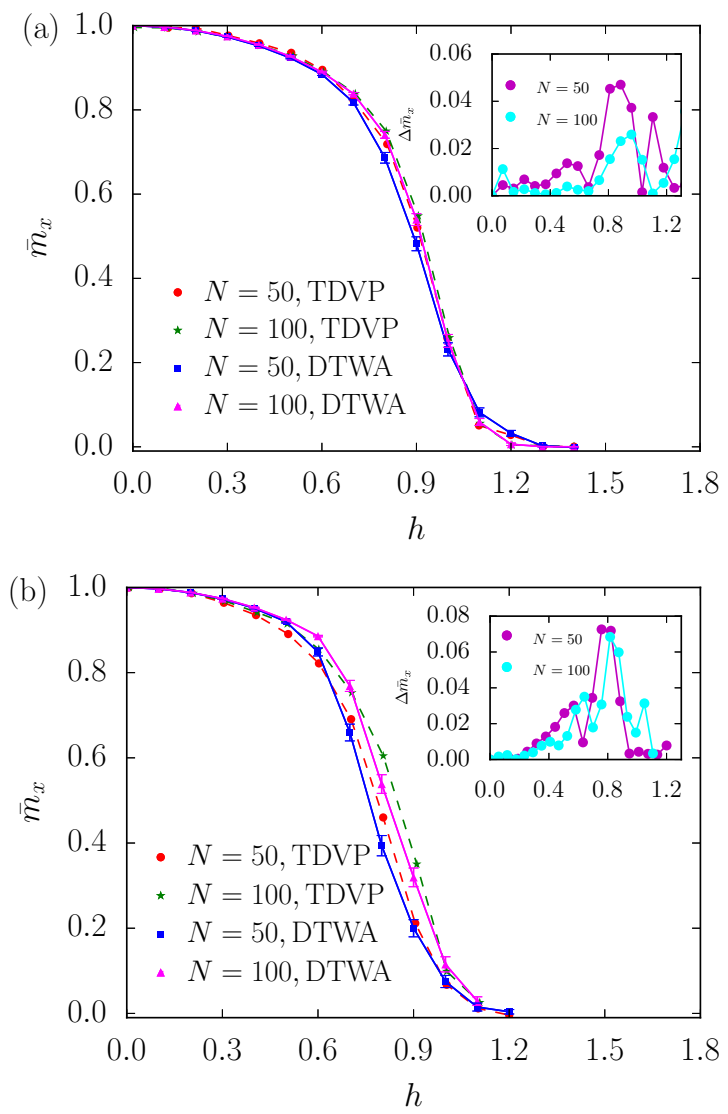

FIG. 3. The long-time average of the magnetization $\bar{m}_{x}$ versus $h$ : Comparison of the results obtained with TDVP [10] and DTWA methods for (a) $\alpha=0.1$ and (b) $\alpha=1.5$. The insets indicate the difference between two methods where $\Delta \bar{m}_{x}=\left|\bar{m}_{x}^{\mathrm{DTWA}}-\bar{m}_{x}^{\mathrm{TDVP}}\right|$.

through TDVP. This gives us an opportunity, because while TDVP can be used for at most $N=200$ (see Ref. [10]), DTWA can be pushed up to much larger sizes, thus offering the possibility for an accurate finite-size scaling.

We conclude this section by comparing DTWA results for the two-dimensional short-range case with the dynamics obtained by means of artificial neural networks (ANNs) [33]. We show an example of comparison in Fig. 4 with data taken from Ref. [33] at large transverse fields in the regime where the Ising symmetry is restored in the long-time limit. As one can see, the DTWA compares remarkably well with the numerically exact ANN data. The idea of ANN approach is to encode the quantum many-body wave function in an ANN [34]. Importantly, ANNs are universal function approximators, which guarantees that the encoding always becomes asymptotically exact in the limit of sufficiently large ANNs. For the curve in Fig. 4, it has been shown that the data has been converged with the size of the neural network; the result is indeed numerically exact.

\section{RESULTS}

In this section, we will illustrate our results for the DPT obtained through the DTWA. We first analyze the onedimensional long-range case [see Eq. (2)]. Later we will analyze the two-dimensional case with short-range interaction,

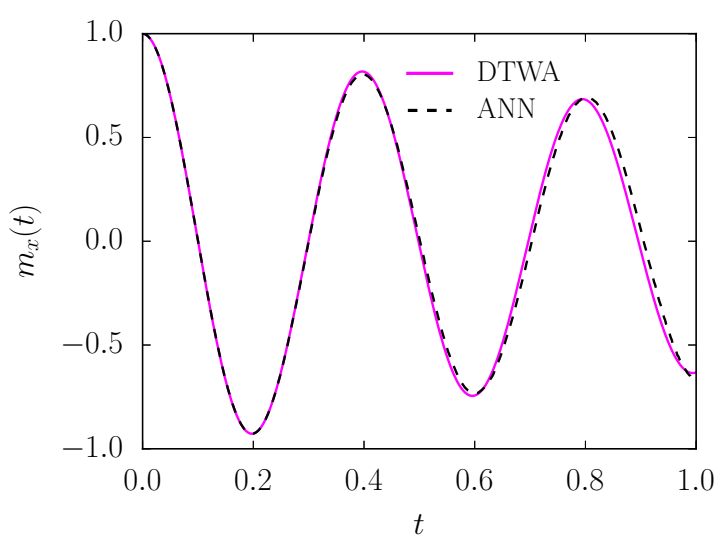

FIG. 4. Comparison of $m_{x}(t)$ obtained by DTWA with the same quantity obtained with ANN [33] for a two-dimensional short-range case. Numerical parameters: $h=8$ and $n_{r}=10000$.

Eq. (3). In this second case, we also use the Binder cumulant to get more reliable indications of the DPT. In both cases, we address the steady-state properties and consider the behavior of the time-averaged magnetization Eq. (6). We consider averages over a time $T$ such that the magnetization has already converged and we specify it in any of the considered cases, explicitly studying the convergence in $T$ for $\alpha \gtrsim 2$.

\section{A. Long-range model in one dimension}

Let us first analyze the one-dimensional long-range case and study the finite-size scaling of $\bar{m}_{x}$ as a function of the transverse field $h$. We start with the case $\alpha=0$, where we can compare DTWA with the exact solution. In Fig. 5, we plot the curves of $\bar{m}_{x}$ versus $h$ for different system sizes $N$, obtained through DTWA (a) and exact diagonalization (b) [35]. There is a good agreement, comparing quantitatively very well, as we show in the inset where we plot $\Delta \bar{m}_{x}=\left|\bar{m}_{x}^{\text {DTWA }}-\bar{m}_{x}^{\text {Exact }}\right|$ versus $h$ for two different values of $N$. As the system size is increased, both have one common crossing point $h_{c}$, making the existence of a phase transition clearly visible. Close to the crossing point, the curves obey a scaling form of the type

$$
\bar{m}_{x, N}(h)=N^{-\beta} f\left[\left(h-h_{c}\right) N^{\delta}\right] .
$$

The possible value $\beta \sim 0$ implies logarithmic corrections of the form $\bar{m}_{x, N}(h) \sim(1 / \ln N) f\left(\left(h-h_{c}\right) N^{\delta}\right)$

We obtain the scaling collapse shown in Fig. 6 (see Appendix $\mathrm{C}$ for details of the scaling procedure). In accordance with the exact solution, the DTWA reproduces the logarithmic corrections (see Fig. 6). Furthermore, we get a scaling exponent $\delta=0.47 \pm 0.04$ in good agreement with the exact exponent $\delta=0.5$ and a critical field $h_{c}=1$ corresponding to the exact result. The data collapse, shown in Fig. 6(b), is excellent. For comparison, the same scaling is shown for the exact diagonalization in Fig. 6(c).

In the following of this section, we are going to apply the methods illustrated here to the case with $\alpha \neq 0$.

We first focus on the values of $\alpha \leqslant 1$. We show some examples of $\bar{m}_{x}$ versus $h$ for different sizes $N$ and different $\alpha$ in Fig. 7 (also in this case the data shown are obtained for $T=200$ where the observables have already attained 

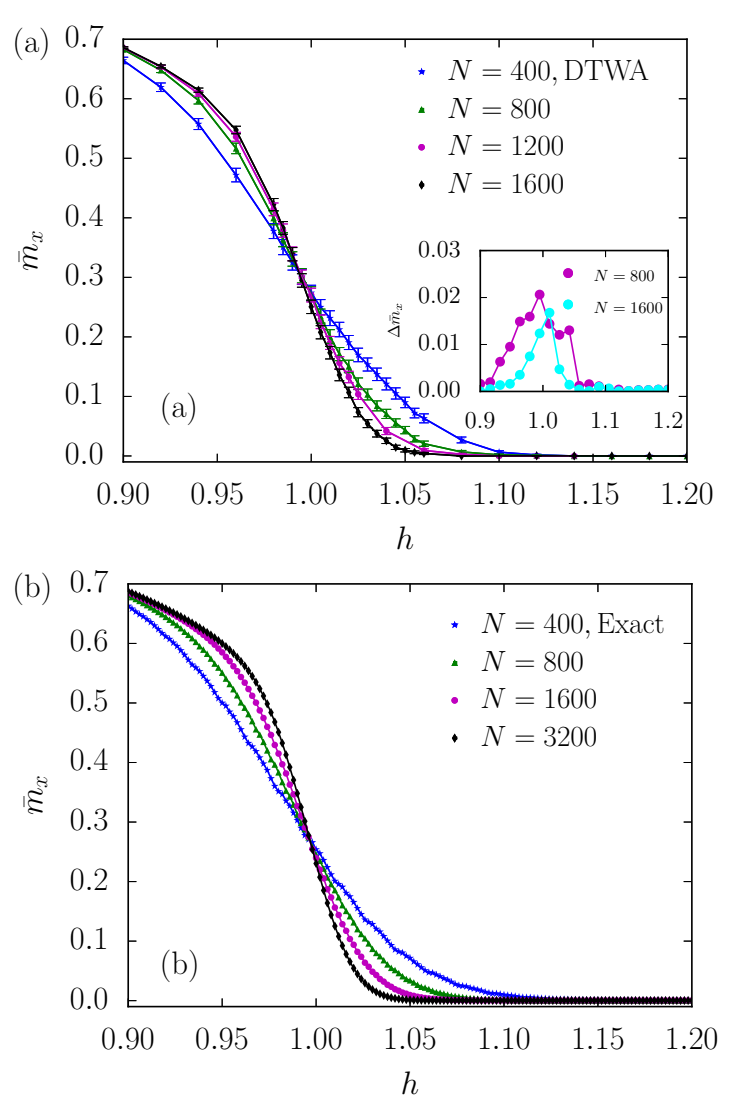

FIG. 5. The long-time average of the magnetization $\bar{m}_{x}$ versus the transverse field $h$ for $\alpha=0$ computed using the DTWA (a) and exact diagonalization (b). There is a good quantitative agreement of the two data sets (see the inset showing $\Delta \bar{m}_{x}=\left|\bar{m}_{x}^{\text {DTWA }}-\bar{m}_{x}^{\text {Exact }}\right|$ versus $h$ for two values of $N$ ). In both panels, we perform the time average over $T=200$.

their stationary value). Before doing the finite-size scaling, let us discuss more qualitatively what happens. For $\alpha=0.1$ [Figs. 7(a) and 7(c)] and $\alpha=0.5$ [Figs. 7(b) and 7(d)], we observe a behavior very similar to the case $\alpha=0$ shown in Fig. 5. In both cases, the curves show a crossing at $h_{c} \sim 1$, the mean-field value. The tiny deviations from the mean-field are not relevant, only due to the fitting procedure. Indeed we can perform a finite-size scaling with the same method used for $\alpha=0$ and with the same scaling function as in Eq. (17). In the same Fig. 7 (lower panel), we show the collapsed curves. For $\alpha \lesssim 0.5$, the critical behavior is mean-field-like. In particular, for $\alpha=0.1$ we find $\delta=0.49 \pm 0.024$ and for $\alpha=0.5$ we find $\delta=0.46 \pm 0.032$.

A different behavior is observed at larger $\alpha$ (shorter-range interactions). We show the data for $\alpha=1$ in Fig. 8. The crossing point is clearly visible albeit the quality of the data collapse is not as good as in the previous cases. Several points are worth being discussed. First, the crossing field is still very close to one. The exponent $\delta=0.76 \pm 0.042$, however, deviates significantly from the mean-field value. Although the DTWA does not allow us to ascertain how sizable the deviation is from the exact scaling analysis, one can be confident in stating that for these parameters $\alpha$ there is still a transition point but the critical behavior deviates from the mean field.
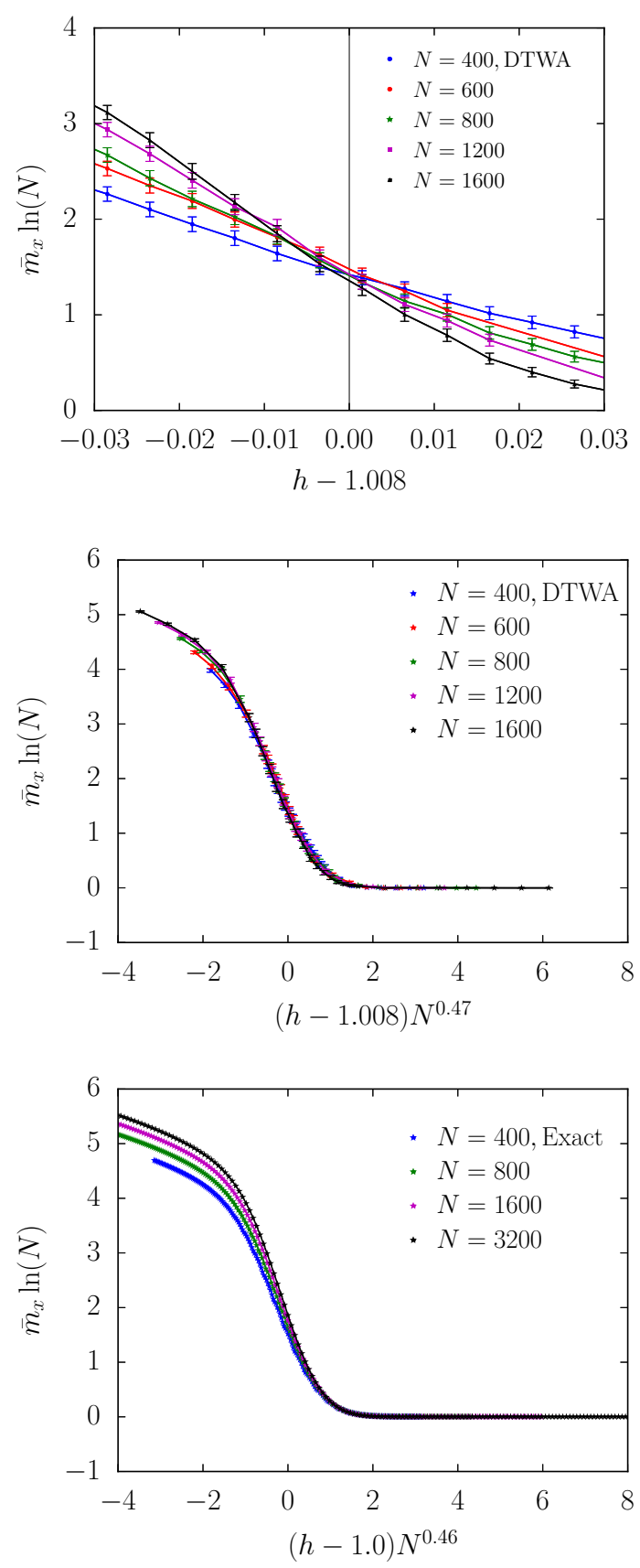

FIG. 6. $(\alpha=0$.) In (a), the data of Fig. 5(b) are magnified around the crossing region to see the details of the crossing region. The imperfect crossing sets an error on the determination of critical field of the order of $10^{-2}$. (b), (c) Figures 5(a) and 5(b) rescaled according to Eq. (17) with the choice of the optimal parameters. The scaling exponents essentially coincide in the two cases.

Another feature that is worth noticing is that there is a range of transverse fields (in the disordered region above the critical field) where the magnetization becomes negative. Our analysis cannot exclude that this "reentrant" behavior might still be a feature of the DTWA approximation, not present in more accurate analysis. It is, however, to be noted that this overshooting of the magnetization might be reminiscent of the chaotic behavior observed in the mean-field dynamics of this model [36]. 

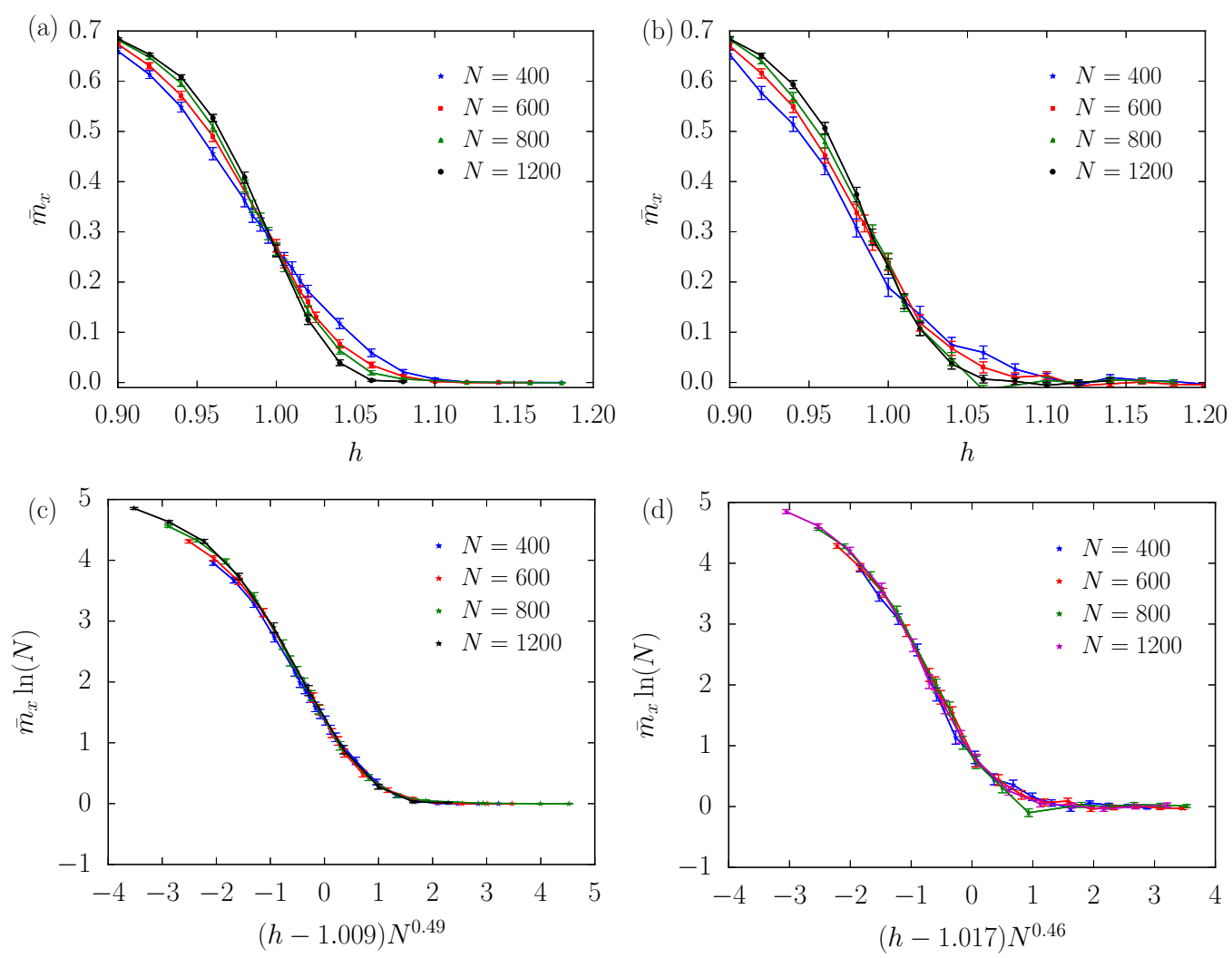

FIG. 7. The long-time average of the magnetization $\bar{m}_{x}$ versus the transverse field $h$ for different values of the range of the interaction: $\alpha=0.1[(\mathrm{a})]$ and $\alpha=0.5$ [(b)]. The scaling collapse is shown in (c) and (d) for $\alpha=0.1,0.5$, respectively. As in the previous figures, $T=200$.

We conclude the analysis of the one-dimensional model by discussing the case of shorter-range interactions, $\alpha \gtrsim 2$. In this regime, DTWA has no more quantitative agreement with the TDVP methods, so the results have only qualitative value. In this regime, we observe that the time-averaged magnetization $\bar{m}_{x}(T)$ decreases with the averaging time $T$ and never reaches a plateau. This behavior can be observed for large enough $h$.

We show these results in Fig. 9. We consider two prototypical cases, $\alpha=2$ [Fig. 9(a)] and $\alpha=3$ [Fig. 9(b)] and we show the time-averaged magnetization $\bar{m}_{x}(T)$ versus $T$ for different values of $h$. For sufficiently large $h$, we see that the average magnetization decreases with $T$ and does not seem to reach a plateau. The corresponding slope of this decrease becomes smaller for smaller values of $h$ and for $h=0.2,0.3$, the decrease is almost invisible (see the insets which are included for illustration). At small values of the field, it would be necessary to go to larger times $T$ to see this trend.

Remarkably, the DTWA gives the same results we have just described for the case of a one-dimensional model with short range interactions, as we discuss in detail in Appendix B. In that case, the model is known to show no long-range order in the excited states [37], and $\bar{m}_{x}$ is doomed to vanish whichever is the value of $h$, as can be shown explicitly in the thermodynamic limit using the JordanWigner transformation [38,39]. Our DTWA numerics suggests that the situation is also the same for the long-range model with $\alpha \gtrsim 2$, but, for sure, this is not a proof and the question is still debated $[10,40]$. From the numerics, obvi- ously, we cannot exclude that a transition point still exists at $h_{c} \ll 1$.

While in one dimension the short-range case is trivial (there is no DPT), the picture changes drastically by moving to higher dimensions. In the next section, we consider the case of a two-dimensional short-range interacting system as defined in Eq. (3).

\section{B. Two-dimensional short-range model}

This case is of particular importance for several reasons. We expect that the transition will deviate from the mean-field behavior. This then leads to the question whether the DTWA is capable of detecting the transition and its non-mean-field type character. If this is the case, a very important question to understand is if the system thermalizes and the dynamical transition corresponds to a thermal-equilibrium transition. The discussion below will try to address some of these points by analyzing both the magnetization and the Binder cumulant.

In Fig. 10, we show the behavior of the time-averaged magnetization as a function of $1 / L$ for different values of the transverse field. Here we take $T=6 \cdot 10^{5}$ due to the long convergence times (this is essentially the limiting factor that forbids us to consider larger lattice sizes). DTWA indicates the existence of a transition for $h^{*} \simeq 0.7$. In the ordered phase, the magnetization increases with the system size and tends to converge only for the largest samples. This type of finite-size effects were also observed in the one-dimensional case where 

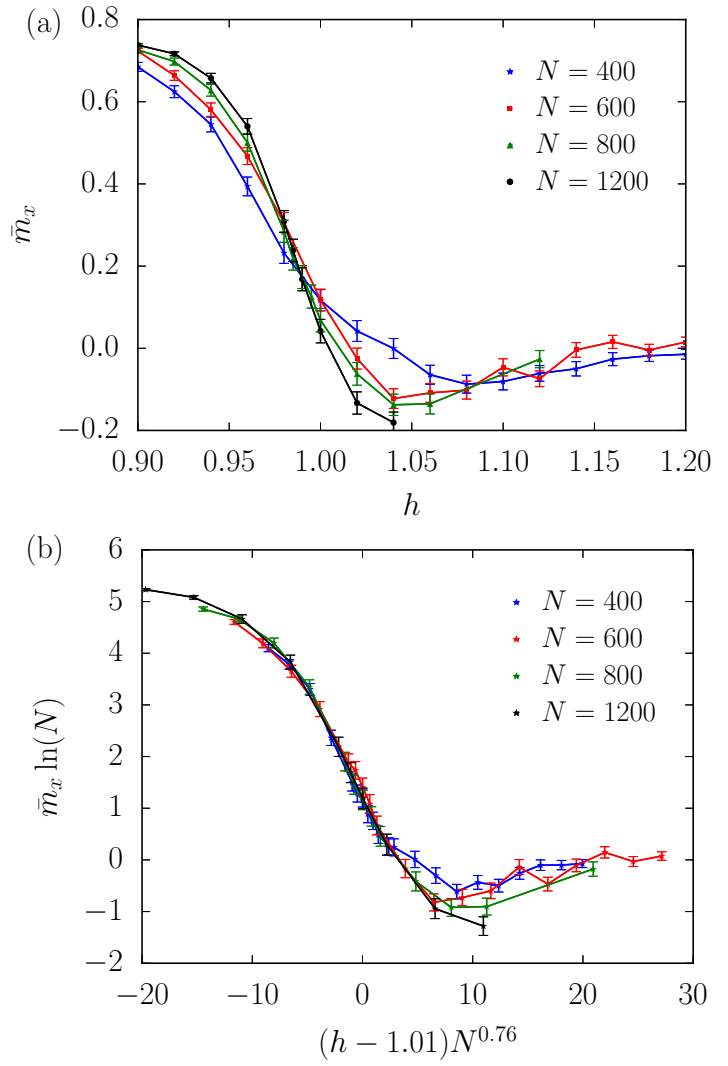

FIG. 8. Scaling plots of the long-time average of the magnetization $\bar{m}_{x}$ versus the transverse field $h$ at $\alpha=1.0$ for different values of $N$. The crossing point is obviously close to $h=1$ (a) but the collapse is not as good as before (b). Not withstanding this limitation in the accuracy of the scaling analysis, the exponents are clearly different from the mean-field values. As in the previous figures, here also $T=200$.

the convergence with size was similarly attained only for $N \sim 100-200$.

We now move to discuss the issue if this transition is the same as the thermal-equilibrium one. First, we notice that the model is quantum chaotic and thermalizing. We can show the presence of quantum chaos by considering the level spacing distribution and checking that it is near to the Wigner-Dyson one [41]. For that purpose, we compute the average level spacing ratio $r$ (see Ref. [42] for a definition and discussion). Using exact diagonalization in the fully symmetric Hilbert subspace of a $5 \times 4$ model, we find a value of $r$ very near to the Wigner-Dyson value $r_{\mathrm{WD}}=0.5295$ for all the considered values of $h$ (see Fig. 11). We therefore expect that the quantum dynamics shows a transition closely corresponding to the thermal one.

We can confirm this expectation by moving to the Binder cumulant analysis. Using this probe, we show that the value of the critical field found with $\bar{m}_{x}$ is not far from the value obtained with quantum Monte Carlo simulations at thermal equilibrium. (We perform the quantum Monte Carlo simulations using the ALPS/looper Library [43-47].) In this framework, we take a temperature $\mathcal{T}(h)$ such that the thermal energy coincides with the value of the energy in the DTWA
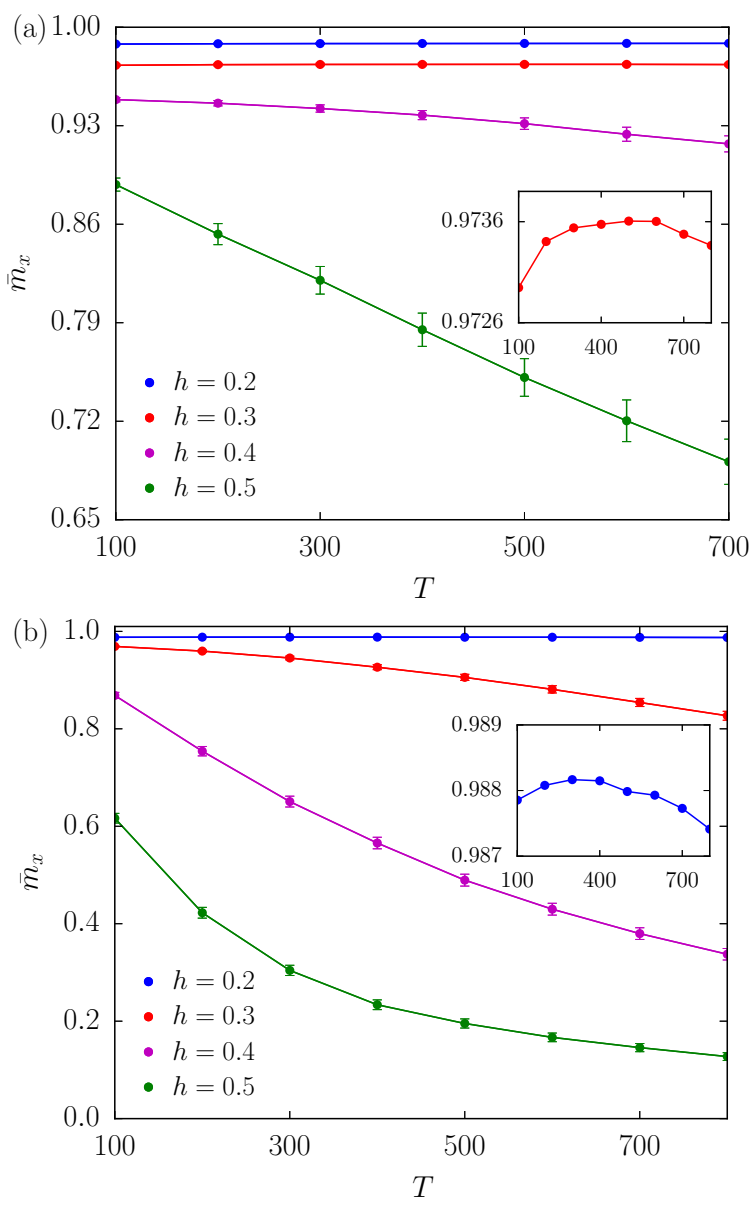

FIG. 9. The long-time average of the magnetization $\bar{m}_{x}$ versus averaging time $T$, (a) and $\alpha=2$ (b) $\alpha=3$. The curves show that the time-averaged magnetization decreases with $T$. This behavior becomes less visible on decreasing $h$, almost disappearing for small $h$. In the insets, a zoom of the curves at $h=0.3$ (a) and $h=0.2$ (b) confirm the same trend. The values $h=0.3$ and $h=0.2$ thus give an upper bound to the possible critical field, as extracted by our analysis. Numerical parameters: $N=100, n_{r}=304$.

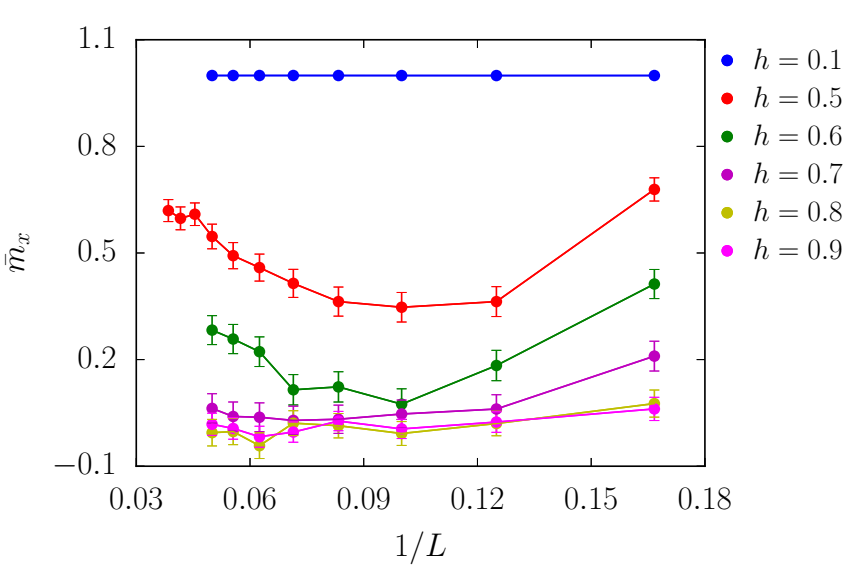

FIG. 10. Results of the DTWA for the long-time average of the magnetization $\bar{m}_{x}$ versus $1 / L$ in a short-range $2 \mathrm{D}$ system for different values of $h$. For $h \gtrsim 0.7$, the magnetization seems to go to zero in the thermodynamic limit. Numerical parameters: $n_{r}=1600, T=$ $6 \cdot 10^{5}$. 


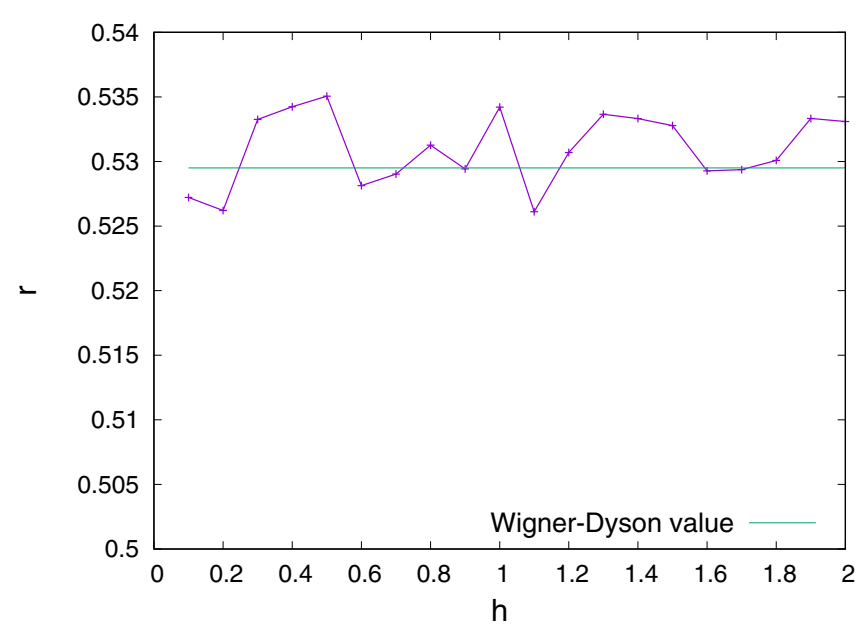

FIG. 11. Level spacing ratio $r$ versus $h$ in the fully symmetric subspace of the two-dimensional model with size $5 \times 4$.

dynamics, and study the properties of the thermal-equilibrium Binder cumulant. It is defined as

$$
U_{L}(\mathcal{T}(h)) \equiv 1-\frac{\left\langle\left[\sum_{i=1}^{N} \hat{\sigma}_{i}^{x}\right]^{4}\right\rangle_{\mathcal{T}(h)}}{3\left\langle\left[\sum_{i=1}^{N} \hat{\sigma}_{i}^{x}\right]^{2}\right\rangle_{\mathcal{T}(h)}^{2}},
$$

where $\langle\cdots\rangle_{\mathcal{T}(h)}$ is the thermal-equilibrium average at the temperature $\mathcal{T}(h)$ defined above. We plot $U_{L}(\mathcal{T}(h))$ versus $h$ for different values of $L$ in Fig. 12. We see that the curves for different system sizes cross each other at $h_{\mathrm{Th}}^{*} \simeq 0.82$. This finding suggests that there is a transition from an ordered to a disordered phase at this value of $h$ (see the general discussion of Ref. [29]). The value of $h_{\mathrm{Th}}^{*}$ is not far from the one we have found studying the magnetization with DTWA, suggesting that this model thermalizes and DTWA can catch up to some extent this aspect of the dynamics.

We find further confirmation of these findings by analyzing with different numerical methods the time-averaged Binder

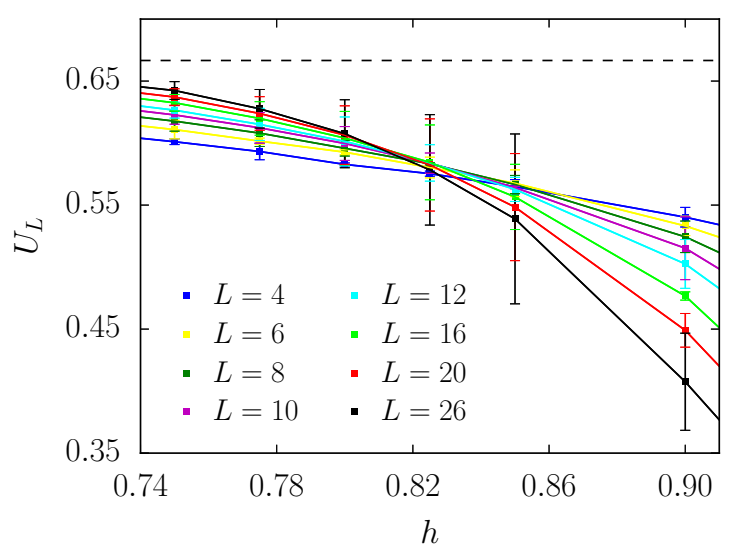

FIG. 12. Binder cumulant at thermal equilibrium obtained via quantum Monte Carlo versus $h$. The considered temperature $\mathcal{T}(h)$ depends on $h$ in such a way that the energy always coincides with the value of the dynamics. Notice the crossing of the curves for different system sizes at $h_{\mathrm{Th}}^{*} \simeq 0.82$. The error bars indicate a worst-case estimate of the error introduced by estimating the temperature at fixed energy $\mathcal{T}(h)$ from numerical data (not a Monte Carlo error).

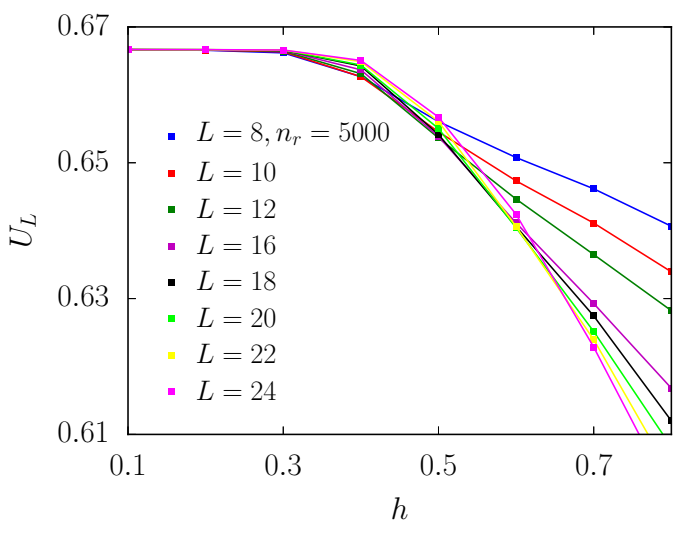

FIG. 13. The Binder cumulant $U_{L}$ [Eq. (7)] versus $h$ in a shortrange 2D system for different values of $h$. Numerical parameters: $T=10^{4}$.

cumulant $U_{L}$, defined in Eq. (7). We study the dynamics with DTWA and exact diagonalization and we consider the behavior of $U_{L}$ versus $h$ for different system sizes. We show data for DTWA in Fig. 13 and the ones for exact diagonalization in Fig. 15. Let us first focus on the DTWA curves in Fig. 13. The crossing between curves at system sizes $L$ and $L+2$ depends on $L$. For the largest sizes we can numerically attain ( $L=22$ ), the crossing occurs at $h^{*} \sim 0.65$. For fields beyond the crossing point, the Binder cumulant rapidly decreases with $L$. This is physically sound: The total magnetization is the sum of the local magnetizations which behave as uncorrelated random variables at large $h$ because the correlation length is very short. The sum of uncorrelated random variables tends to a Gaussian as the number of random variables increases and for a Gaussian the Binder cumulant vanishes. For small values of $h$, on the opposite, $U_{L}$ increases with $L$. Therefore, a crossing point between curves for different $L$ appears.

The Binder cumulant has been evaluated averaging over a time $\left(T=10^{4}\right)$ shorter than the time needed to attain an asymptotic value in the DTWA scheme. The point is that, before this asymptotic value, the Binder cumulant attains a metastable plateau in the DTWA scheme: We show some

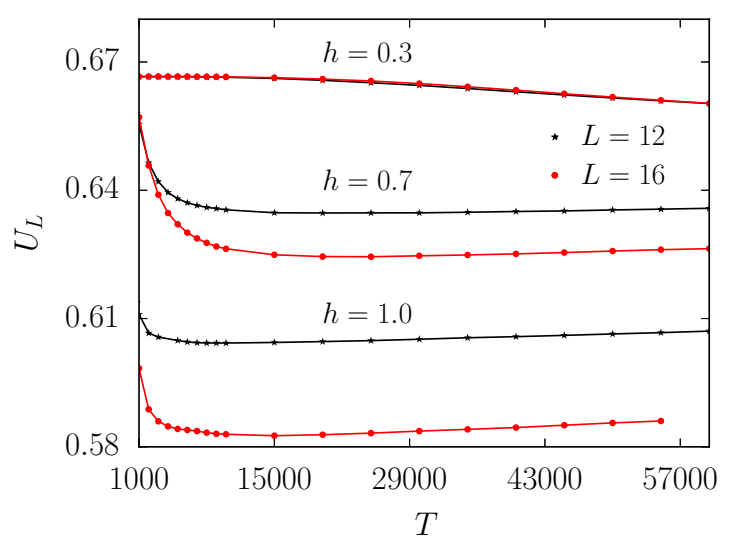

FIG. 14. The Binder cumulant $U_{L}$ [Eq. (7)] versus $T$ in a short range $2 \mathrm{D}$ system for different values of $h$ and $L$. Notice the metastable plateau. Numerical parameters: $T=10^{4}$. 


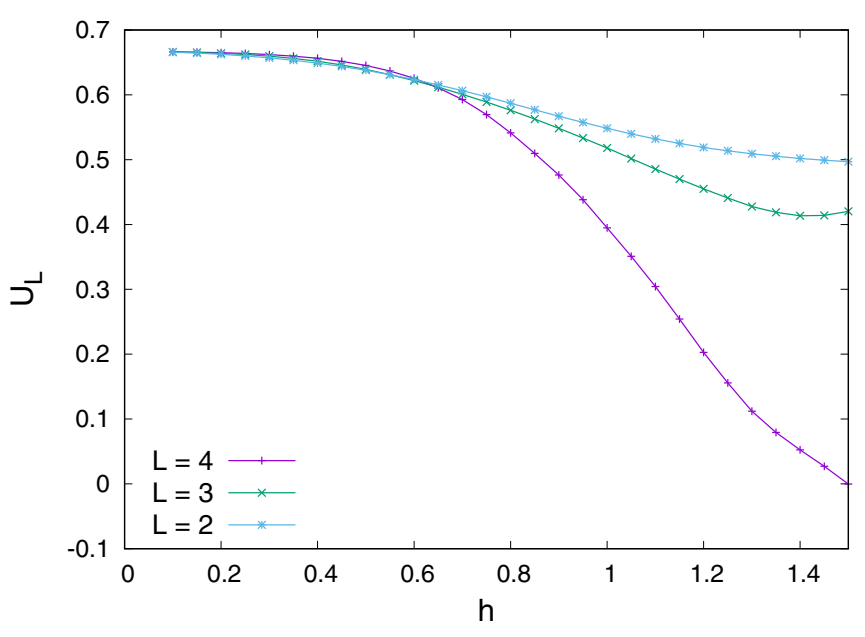

FIG. 15. Exact diagonalization result of the Binder cumulant $U_{L}$ [Eq. (7)] versus $h$ in a short-range 2D system for different values of $L$. Numerical parameters: $T=1000$.

examples in Fig. 14. This plateau gives rise to the crossing behavior we can see in Fig. 13 while the asymptotic value does not. The metastable plateau therefore shows a behavior more similar to the ones given by quantum Monte Carlo (Fig. 12) and by exact diagonalization (Fig. 15). This suggests that, in this context, DTWA gives physically more sound results for a finite time, although the difference between the metastable plateau and the asymptotic value is very small. We remark that this plateau is an effect of the approximation and does not correspond to any prethermalization behavior in the actual physics.

We also study the behavior of the Binder cumulant by means of exact diagonalization. In Fig. 15, we show the exactdiagonalization Binder cumulant versus $h$ for small system sizes. The trend is the same as that observed in Fig. 13. The crossing occurs around $h^{*} \sim 0.6$, which is in good agreement with the value found using DTWA. We stress again that for increasing system size the Binder cumulant tends to $2 / 3$ in the ordered phase and to zero in the disordered one, exactly as it occurs in the thermal-equilibrium case.

In conclusion, for the two-dimensional short-range case, there is a dynamical transition closely corresponding to the thermal one due to the fact that the system appears to be quantum chaotic and thermalizing. Remarkably, DTWA can see the existence of this transition.

\section{CONCLUSIONS}

In conclusion, we have used DTWA to study the dynamical quantum phase transition in Ising spin models. Our aim was exploring the existence of a transition between an ordered and a disordered phase in the steady state and the properties of this transition focusing on a local order parameter, the timeaveraged longitudinal magnetization.

We have first focused on the long-range one-dimensional case where interactions decay with the power $\alpha$ of the distance. Here we have compared DTWA with numerically exact results (exact diagonalization for $\alpha=0$ and TDVP) and we have found good agreement. Thanks to the good scalability of DTWA, we have done a finite-size scaling of the time- averaged longitudinal magnetization and we have studied the critical exponents of the transition between ordered and disordered phase. For $\alpha$ small ( $\alpha=0.1,0.5)$, we have found the same critical exponents as the mean-field case $(\alpha=0)$. For $\alpha=1$, we have found critical exponents significantly different from the mean-field case and we have found that the magnetization changes sign in the critical region. We do not know if this is a physical result or an effect of the DTWA approximation which should not work very well in the critical region due to the long-range correlations of the physical system. For $\alpha \gtrsim 2$, we have found no scaling at all with the system size and we have put a lower bound to the value of $h$ for which the longitudinal magnetization vanishes at long times. We argue that this is most probably the case also for smaller $h$ but we cannot see it due to the extremely long convergence times in the DTWA scheme (this is the same situation occurring if we apply DTWA to a short-range one-dimensional Ising model).

We further considered the two-dimensional short-range model, not considered in this context so far, again applying the DTWA approximation. Our data confirm that the DTWA is able to capture the existence of a transition and the value of the critical field compares well with the one of a corresponding thermal transition. We argued that this is physically sound showing that the model is quantum chaotic by means of exact diagonalization. To attempt a scaling analysis and thus to confirm that the associated critical exponents are the thermal ones, it would be necessary to consider even larger system sizes, which might be an interesting prospect for the future.

Our work can also be considered as a contribution toward the clarification of the range and the limitations of qualitative and quantitative applicability of the DTWA.

\section{ACKNOWLEDGMENTS}

We would like to acknowledge fruitful discussions with Silvia Pappalardi. R.F. acknowledges financial support from the Google Quantum Research Award. R.K. acknowledges financial support from the ICTP STEP program. M.S. was supported through the Leopoldina Fellowship Programme of the German National Academy of Sciences Leopoldina (LPDS 2018-07) with additional support from the Simons Foundation. This project has received funding from the European Research Council (ERC) under the European Union's Horizon 2020 research and innovation program (Grant Agreement No. 853443), and M.H. further acknowledges support by the Deutsche Forschungsgemeinschaft via the Gottfried Wilhelm Leibniz Prize program.

\section{APPENDIX A: DTWA SAMPLING}

As discussed in Sec. III, in the DTWA approach, one has to solve classical equations of motions for different random initial configuration. Physical quantities are obtained upon averaging over this initial distribution. In this Appendix, we report on some details of the sampling procedure we used to obtain the results reported in the body of the paper.

First, it is important to understand how the results depend on the number of random initial realizations $n_{r}$. In Fig. 16, we consider the dependence of the average magnetization as 


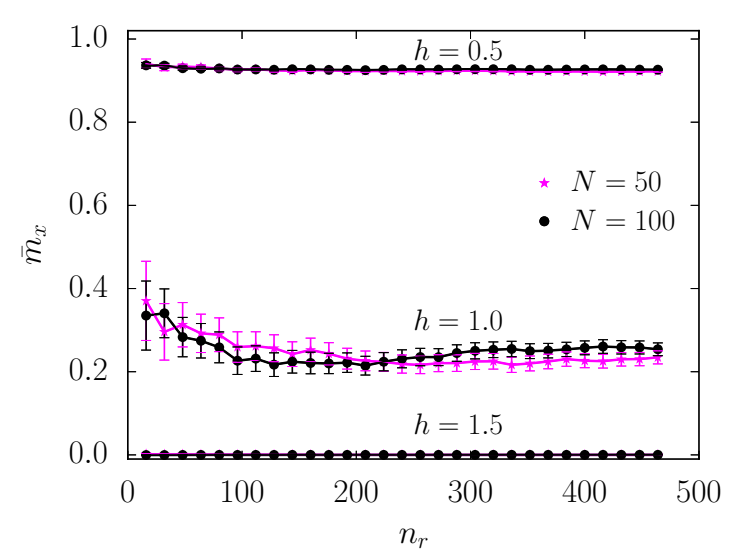

FIG. 16. The long-time average of the magnetization $\overline{m_{x}}$ versus number of realizations $n_{r}$ for three different values of the transverse field. The convergence changes depending on the distance from the critical point. However, in all the shown cases, averaging over $100-200$ configurations already guarantees that the obtained result is reliable. In the case shown here, $\alpha=0.1$. We tested that this behavior is quite generic.

a function of the number of initializations $n_{r}$. We show the case of $\alpha=0.1$; the behavior is, however, quite generic. Away from the critical field $h_{c}$, the order parameter $\bar{m}_{x}$ converges
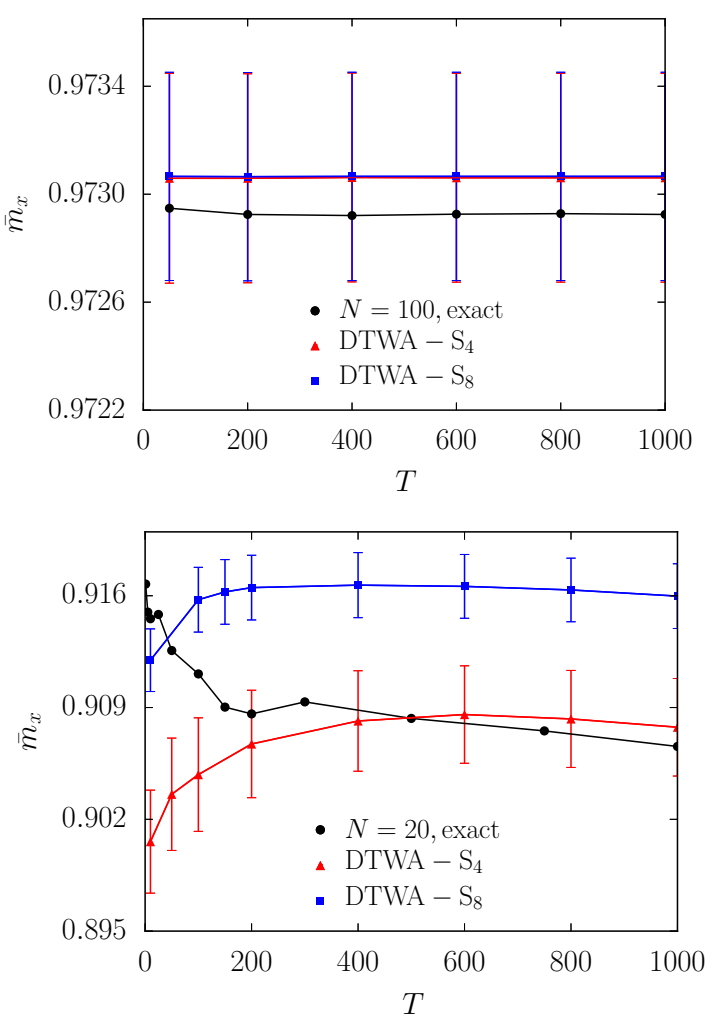

FIG. 17. The time average of the magnetization $\bar{m}_{x}$ versus the averaging time $T$ : Comparison of the results obtained with exact diagonalization and different sampling schemes of DTWA (the sampling schemes $S_{4}$ and $S_{8}$ are defined and discussed in Ref. [48]). We consider $\alpha=0.0, N=100$ (upper panel), and $\alpha=1.0, N=20$ (lower panel). Other parameters: $h=0.32, n_{r}=2000$. very rapidly to its asymptotic value and no significant changes happen by increasing $n_{r}$. Since we are interested in determining transition points, the behavior $\bar{m}_{x}$ as a function of $n_{r}$ is more notable in the critical region. Close to the transition point, the convergence with the number of realizations is slower. In any case, after a few hundreds of initial configurations the results seem stable. We choose $n_{r}=504$ for most of the calculations, if not stated otherwise.

In addition to the number of initial configurations over which performing the sampling, another aspect to consider is the choice of the sampling scheme. Indeed, using phase point operator $\hat{A}_{\alpha}$, one can map each basis state of Hilbert space to a point in phase space. There are different possible choices of this phase operator and the one shown in Eq. (8) is not the only one. Any other possible choice for phase operator can be derived by some unitary transformation, $\hat{A}_{\beta}^{\prime}=\hat{U} \hat{A}_{\beta} \hat{U}^{\dagger}$.
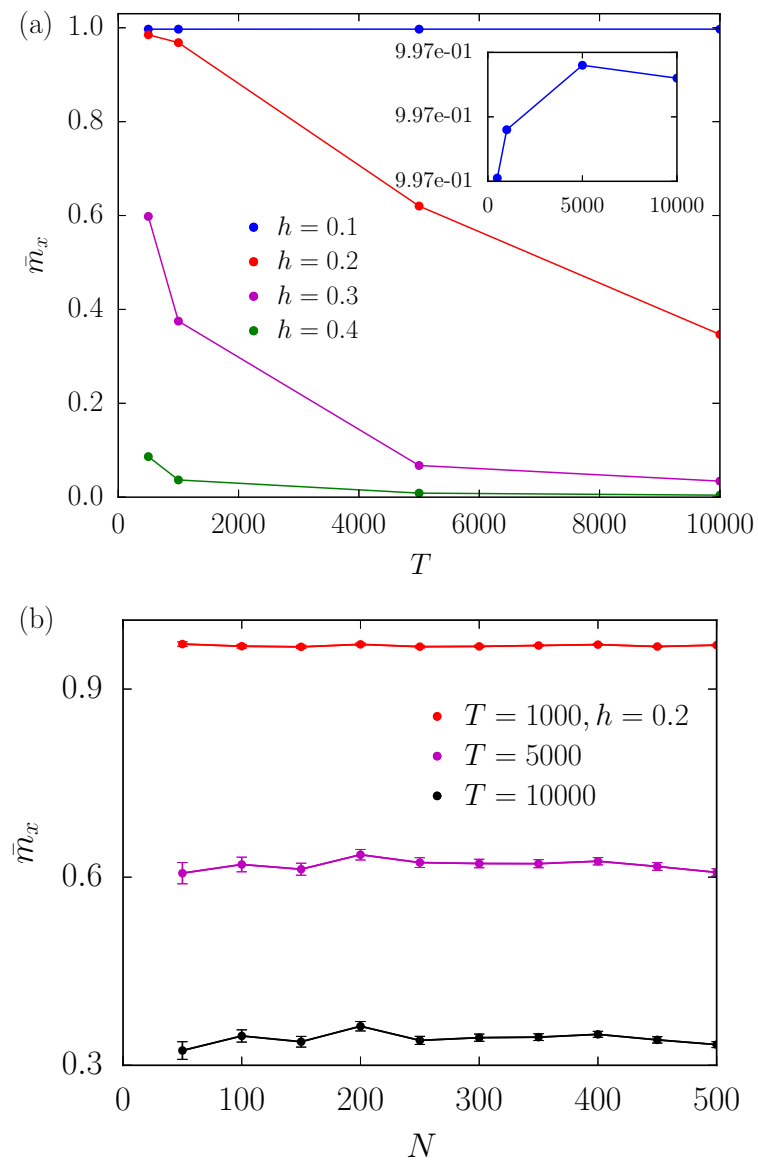

FIG. 18. Top panel: The time-average of the magnetization $\bar{m}_{x}(T)$ versus averaging time $T$ in the Ising chain with short-range interaction. For larger $h$, the average magnetization decreases with $T$ toward 0 without ever reaching a plateau: This suggests that $\bar{m}_{x}(T) \stackrel{T \rightarrow \infty}{\rightarrow} 0$ as in the actual physics. For $h=0.1$, this decay behavior can only be seen very slightly (inset), but this is an artifact of the DTWA and not a physical effect. Numerical parameters: $N=100$, $n_{r}=304$. Bottom panel: Plot of the magnetization versus the system size $N$. The correlations are very short range for this model and this is reflected in the insensitivity on $N$ of the average. 

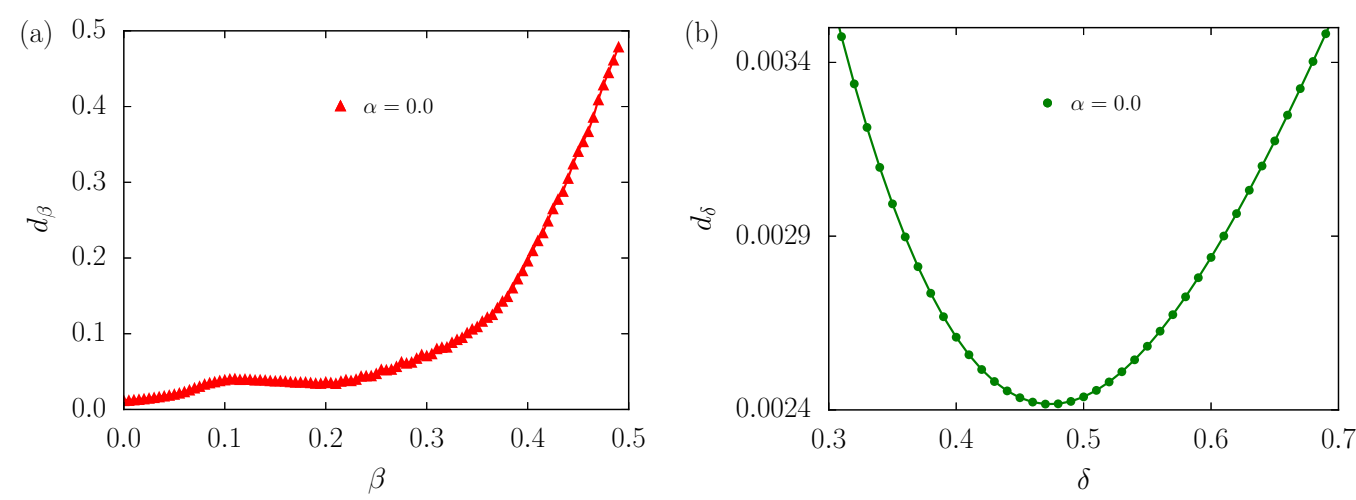

FIG. 19. (a) Minimum distance between curves in Fig. 5(a) as a function of $\beta$. (b) Cost function as function of $\delta$. Here $\alpha=0.0$.

In Ref. [48], the following phase operator was considered (more details about this construction can be found there):

$$
\hat{A}_{\beta}^{\prime}=\frac{\mathbf{1}+\mathbf{s}_{\beta}^{\prime} \cdot \hat{\boldsymbol{\sigma}},}{2}
$$

where $\boldsymbol{s}_{\beta}^{\prime}$ can take the values $\left(\begin{array}{lll}1-1 & 1\end{array}\right),(-1-1-1)$, $\left(\begin{array}{lll}1 & 1 & -1\end{array}\right)$, and $\left(\begin{array}{lll}-1 & 1 & 1\end{array}\right)$ and $\hat{\sigma}=\left(\begin{array}{lll}\hat{\sigma}^{x} & \hat{\sigma}^{y} & \hat{\sigma}^{z}\end{array}\right)$ which is obtained by flipping the sign of the second component of $\boldsymbol{s}_{\beta}$.

Figure 17 shows the comparison, as a function of the averaging time $T$ for two different values of $\alpha$. In the same figure, DTWA is further compared to exact diagonalization. In the fully connected case $(\alpha=0)$, the different samplings lead to essentially the same result and agree with the exact-diagonalization data. Smaller distances are observed in the bottom panel for the case $\alpha=1$. It should be noted that deviations appear only at the the third decimal digit. These differences may be important only very close to the transition point and may also contribute to the uncertainties in the scaling plots that we observe for $\alpha \sim 1$. However, the analysis of the present work does not depend on the sampling scheme.

\section{APPENDIX B: SHORT-RANGE MODEL IN ONE DIMENSION}

In the case of spin chain with short-range interaction, there is no ordered nonequilibrium steady state [37-39] (it corresponds to the long-range one-dimensional model studied in the limit of very large $\alpha$ ). It is useful to check this result with DTWA as an additional test of its quality. Following the same approach used to argue the absence of a critical point for $\alpha \gtrsim 2$, we analyze how the magnetization scales with $T$ for different values of the transverse field. The result of this analysis is presented in Fig. 18. Down to $h=0.1$, the steadystate magnetization (at large $T$ ) tends to zero (top panel). The inset in the top panel shows that to see the suppression of the magnetization at large $T$, one should go to very large values. In the top panel, we considered a chain of length $N=100$. Because of the short-range correlations in this case, the behavior is essentially independent of $N$ as displayed by the bottom panel of Fig. 18.

\section{APPENDIX C: DETERMINATION OF THE CRITICAL EXPONENTS}

To determine the best approximations to $h_{c}$ and to the exponent $\beta$, we find the values of $h$ and $\beta$ such that the distance function between the average magnetization curves at different $N$,

$$
d_{\beta}(h)=\sum_{N, N^{\prime}<N}\left|N^{\beta} \bar{m}_{x, N}(h)-N^{\prime \beta} \bar{m}_{x, N^{\prime}}(h)\right|,
$$

is minimum. In this way, we find $h_{c}=1.008 \pm 0.01$, a value very near to the exact one $h_{c}=1$. Moreover, we find $\beta=0$, as we can see in Fig. 19, but we scale the average magnetization with $\ln N$ in order to take into account the logarithmic corrections. For finding the optimal $\delta$, we minimize with respect to $\delta$ the cost function

$D_{\delta}=\frac{\sum_{N^{\prime}, N<N^{\prime}} \int \mathrm{d} x\left[\bar{m}_{x, N}\left(h_{c}+N^{-\delta} x\right)-\bar{m}_{x, N^{\prime}}\left(h_{c}+N^{\prime-\delta} x\right)\right]^{2}}{\sum_{N^{\prime}, N<N^{\prime}} \int \mathrm{d} x\left[\bar{m}_{x, N}^{2}\left(h_{c}+N^{-\delta} x\right)+\bar{m}_{x, N^{\prime}}^{2}\left(h_{c}+N^{\prime-\delta} x\right)\right]}$.

The errorbars in $\delta$ are evaluated in the following way. If we have to perform our minimization procedure on a set of $K$ data curves, we consider all the $K$ distinct subsets of $K-1$ curves. In each of these subsets, we perform the minimization procedure and then we get $K$ different values of $\delta$. The standard deviation of these $K$ values of $\delta$ provides the errorbar. In Fig. 19, we consider in detail an example of application of our method. In Fig. 19(a), we show the minimum distance versus $\beta$, while in Fig. 19(b) we show the cost function versus $\delta$ for different $\alpha$ and $h_{c}$ found using the logarithmic scaling [see below, Eq. (17)]. To perform the integration, we apply a cubic spline interpolation. The dependence of $D_{\delta}$ on $\delta$ is shown in Fig. 19(b); we find the minimum in $\delta=0.47$, as we have elucidated in the main text.
[1] A. Polkovnikov, K. Sengupta, A. Silva, and M. Vengalattore, Rev. Mod. Phys. 83, 863 (2011).
[2] I. Bloch, J. Dalibard, and W. Zwerger, Rev. Mod. Phys. 80, 885 (2008). 
[3] D. A. Abanin, E. Altman, I. Bloch, and M. Serbyn, Rev. Mod. Phys. 91, 021001 (2019).

[4] J. Eisert, M. Friesdorf, and C. Gogolin, Nat. Phys. 11, 124 (2015).

[5] M. Heyl, Rep. Prog. Phys. 81, 054001 (2018).

[6] V. Khemani, R. Moessner, and S. L. Sondhi, arXiv:1910.10745 [cond-mat.str-el].

[7] E. Altman, K. R. Brown, G. Carleo, L. D. Carr, E. Demler, C. Chin, B. DeMarco, S. E. Economou, M. A. Eriksson, K.-M. C. Fu, M. Greiner, K. R. A. Hazzard, R. G. Hulet, A. J. Kollar, B. L. Lev, M. D. Lukin, R. Ma, X. Mi, S. Misra, C. Monroe, K. Murch, Z. Nazario, K.-K. Ni, A. C. Potter, P. Roushan, M. Saffman, M. Schleier-Smith, I. Siddiqi, R. Simmonds, M. Singh, I. B. Spielman, K. Temme, D. S. Weiss, J. Vuckovic, V. Vuletic, J. Ye, and M. Zwierlein, arXiv: 1912.06938.

[8] B. Sciolla and G. Biroli, Phys. Rev. Lett. 105, 220401 (2010).

[9] B. Sciolla and G. Biroli, J. Stat. Mech. (2011) P11003.

[10] B. Žunkovič, M. Heyl, M. Knap, and A. Silva, Phys. Rev. Lett. 120, 130601 (2018).

[11] D. A. Huse, R. Nandkishore, V. Oganesyan, A. Pal, and S. L. Sondhi, Phys. Rev. B 88, 014206 (2013).

[12] G. Piccitto and A. Silva, J. Stat. Mech. (2019) 094017.

[13] A. Lerose, B. Žunkovič, J. Marino, A. Gambassi, and A. Silva, Phys. Rev. B 99, 045128 (2019).

[14] S. Pappalardi, A. Russomanno, B. Žunkovič, F. Iemini, A. Silva, and R. Fazio, Phys. Rev. B 98, 134303 (2018).

[15] R. M. Nandkishore and S. L. Sondhi, Phys. Rev. X 7, 041021 (2017).

[16] S. Nag and A. Garg, Phys. Rev. B 99, 224203 (2019).

[17] F. Liu, R. Lundgren, P. Titum, G. Pagano, J. Zhang, C. Monroe, and A. V. Gorshkov, Phys. Rev. Lett. 122, 150601 (2019).

[18] A. Y. Guo, M. C. Tran, A. M. Childs, A. V. Gorshkov, and Z.-X. Gong, arXiv:1906.02662 [quant-ph].

[19] A. Lerose, B. Žunkovič, A. Silva, and A. Gambassi, Phys. Rev. B 99, 121112(R) (2019).

[20] R. Verdel, F. Liu, S. Whitsitt, A. V. Gorshkov, and M. Heyl, arXiv:1911.11382 [cond-mat.stat-mech].

[21] M. Schiró and M. Fabrizio, Phys. Rev. Lett. 105, 076401 (2010).

[22] B. Neyenhuis, J. Zhang, P. W. Hess, J. Smith, A. C. Lee, P. Richerme, Z.-X. Gong, A. V. Gorshkov, and C. Monroe, Sci. Adv. 3, e1700672 (2017).

[23] J. Zhang, G. Pagano, P. W. Hess, A. Kyprianidis, P. Becker, H. Kaplan, A. V. Gorshkov, Z. X. Gong, and C. Monroe, Nature (London) 551, 601 (2017).

[24] J. Zhang, P. W. Hess, A. Kyprianidis, P. Becker, A. Lee, J. Smith, G. Pagano, I. D. Potirniche, A. C. Potter, A. Vishwanath, N. Y. Yao, and C. Monroe, Nature (London) 543, 217 (2017).

[25] J. Vidal, G. Palacios, and C. Aslangul, Phys. Rev. A 70, 062304 (2004).

[26] W. K. Wootters, Ann. Phys. 176, 1 (1987).
[27] J. Schachenmayer, A. Pikovski, and A. M. Rey, Phys. Rev. X 5, 011022 (2015).

[28] M. Kac, J. Math. Phys. (NY) 4, 216 (1963).

[29] K. Binder, Z. Phys. B 43, 119 (1981).

[30] A. Polkovnikov, Ann. Phys. 325, 1790 (2010).

[31] J. Haegeman, C. Lubich, I. Oseledets, B. Vandereycken, and F. Verstraete, Phys. Rev. B 94, 165116 (2016).

[32] A. Russomanno, R. Fazio, and G. E. Santoro, Europhys. Lett. 110, 37005 (2015).

[33] M. Schmitt and M. Heyl, arXiv:1912.08828 [cond-mat.str-el].

[34] G. Carleo, and M. Troyer, Science 355, 602 (2017).

[35] Exact diagonalization is performed, exploiting the fact that the model for $\alpha=0$ conserves the total-spin operator and one can restrict to the subspace with total spin $N / 2$. For details, see, for instance, Ref. [32].

[36] G. Piccitto, B. Žunkovič, and A. Silva, Phys. Rev. B 100, 180402(R) (2019).

[37] G. G. Batrouni, and R. T. Scalettar, Quantum phase transitions, in Ultracold Gases and Quantum Information, Lecture Notes of the Les Houches Summer School in Singapore, (Oxford University Press, New York, 2011), Vol. 91.

[38] S. Suzuki, J.-I. Inoue, and B. K. Chakrabarti, Quantum Ising Phases and Transitions in Transverse Ising Models, Lecture Notes in Physics (Springer, Berlin, 2012), Vol. 862.

[39] S. Pappalardi, A. Russomanno, A. Silva, and R. Fazio, J. Stat. Mech. (2016) 053104.

[40] J. C. Halimeh, V. Zauner-Stauber, I. P. McCulloch, I. de Vega, U. Schollwöck, and M. Kastner, Phys. Rev. B 95, 024302 (2017).

[41] F. Haake, Quantum Signatures of Chaos (Springer, Berlin, 2001).

[42] V. Oganesyan and D. A. Huse, Phys. Rev. B 75, 155111 (2007).

[43] The ALPS project, http://alps.comp-phys.org/.

[44] The ALPS/looper project, http://wistaria.comp-phys.org/alpslooper/.

[45] S. Todo and K. Kato, Phys. Rev. Lett. 87, 047203 (2001).

[46] A. Albuquerque, F. Alet, P. Corboz, P. Dayal, A. Feiguin, S. Fuchs, L. Gamper, E. Gull, S. Gürtler, A. Honecker, R. Igarashi, M. Körner, A. Kozhevnikov, A. Läuchli, S. Manmana, M. Matsumoto, I. McCulloch, F. Michel, R. Noack, G. Pawłowski, L. Pollet, T. Pruschke, U. Schollwöck, S. Todo, S. Trebst, M. Troyer, P. Werner, and S. Wessel, J. Magn. Magn. Mater. 310, 1187 (2007).

[47] B. Bauer, L. D. Carr, H. G. Evertz, A. Feiguin, J. Freire, S. Fuchs, L. Gamper, J. Gukelberger, E. Gull, S. Guertler, A. Hehn, R. Igarashi, S. V. Isakov, D. Koop, P. N. Ma, P. Mates, H. Matsuo, O. Parcollet, G. Pawłowski, J. D. Picon, L. Pollet, E. Santos, V. W. Scarola, U. Schollwöck, C. Silva, B. Surer, S. Todo, S. Trebst, M. Troyer, M. L. Wall, P. Werner, and S. Wessel, J. Stat. Mech. (2011) P05001.

[48] S. Czischek, M. Gärttner, M. Oberthaler, M. Kastner, and T. Gasenzer, Quantum Sci. Technol. 4, 014006 (2018). 